\title{
Spacial Distribution of Salinity and the Mechanism of Saltwater Intru- sion in the Modaomen Water Channel of Pear River Estuary
}

\author{
J. B. $\mathrm{Liu}^{1, *}, \mathrm{Y} . \mathrm{Bao}^{2}$ \\ ${ }^{1}$ Laboratory of Environmental Mechanics, Institute of Mechanics, Chinese Academy of Sciences, Beijing 100190, China \\ ${ }^{2}$ Department of Applied Mechanics and Engineering School of Engineering, Sun Yat-sen University, Guangzhou 510275, \\ China
}

Email: liujiebin@imech.ac.cn

\begin{abstract}
Modaomen channel is an important fresh water resource in Pearl River Delta. It has been impacted by saltwater intrusion frequently in the last decade. This has drawn more and more attention from scientists and engineers. The hydrodynamic mechanism of saltwater intrusion is still impercipient. In the present paper, hydrographs of velocity and salinity in the channel are analyzed based on field observations of velocity and salinity of upper, middle, and lower water layers at several stations along the Modaomen channel. It is found that the transport of salinity in Modaomen channel is obviously different from other estuaries. As the tidal range increases from neap to spring tide, the salinity in each water layer decreases unexpectedly. This peculiar phenomenon is attributed to the extraordinary flow process in the channel. When salinity value in each layer and vertical salinity gradient are lower during spring tide, no matter on rising or ebbing tide, the flow velocity monotonously decreases from water surface to the bottom, which is suggested by common sense. However, when salinity values and vertical salinity gradient are higher during neap tide, the flow velocity unexpectedly increases from water surface to the bottom during flood period, and flood duration of the bottom current is surprisingly as long as 15-18 hours. In addition, an inflexional velocity profile may remain amazingly for about 9 hours. This could be driven by the baroclinic pressure under the condition of tides, topography and upstream runoff discharge of this channel.
\end{abstract}

Key words: Modaomen channel, Pear River estuary, distribution of salinity, saltwater intrusion

\section{INTRODUCTION}

Salt water intrusion is a very common phenomenon among estuaries. Modaomen channel, which serves as an important fresh water resource in Pearl River Delta Region, has been impacted by saltwater intrusion frequently during the dry season in the last decades. This has drawn more and more attention from scientists and engineers for its special performance. To learn the salinity activity is of significance for fresh water supply and understanding of estuarine process.

The time series of salinity intrusion length, defined as the distance from the river mouth to the salt intrusion limit where the surface $0.5 \mathrm{ppt}$ salinity is, was observed and studied [1]. The salinity intrusion is always enhanced suddenly in two days during neap tide. The intrusion length reaches its maximum in a few days. The saline front goes upstream and downstream near the maximum for several days. As the tidal range increases, the saline front moves seawards rather than landwards. This unusual phenomenon may account for the fact that the salinity intrusion event is serious during neap tide.

We analyzed the saline front motions in dry year and wet year [2]. The upstream runoff discharge only has an influence on the intrusion speed and intrusion length, but does not change the periodicity of salinity intrusion in Modaomen channel. From the time series of salinity intrusion length, the intrusion speed in the two days when the salinity intrusion enhanced suddenly is the key factors determining the strength of salinity intrusion. 
In the present paper, we investigate this special phenomenon in Modaomen by using field observations of velocity and salinity of upper, middle, and lower water layers $(0.2 d, 0.6 d, 0.8 d$ respectively) at several stations along the Modaomen channel. The typical stations selected along the channel is Zhuyin station (Figure 1)

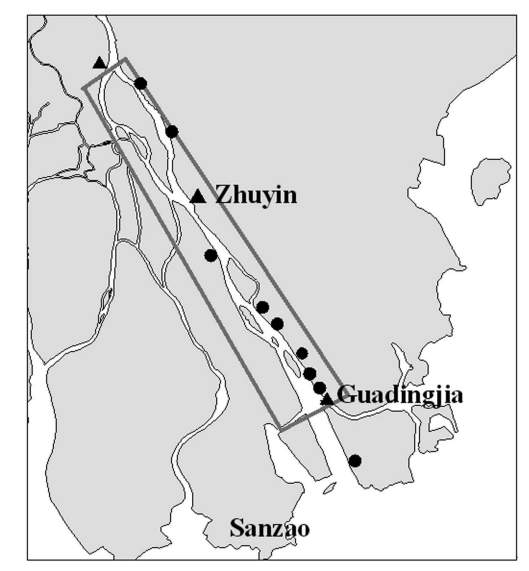

Figure 1: The Modaomen estuary and hydrological stations along it

\section{ANALYSIS OF HYDROGRAPHS OF VELOCITY AND SALINITY AT TYPICAL STATIONS}

Figure 2 shows the time series of velocity and salinity at Zhuyin station. The smooth curve denotes the history of tide level at Sanzao station (Figure 1). The others denote time series of velocity and salinity in each water layer respectively, as shown in Figure 2. It can be seen that as the tidal range increases from neap to spring tide (from December 10/2007 to December 16), the salinity in each water layer decreases unexpectedly. This is very unusual among other estuaries. This phenomenon must be associated with the flow process.
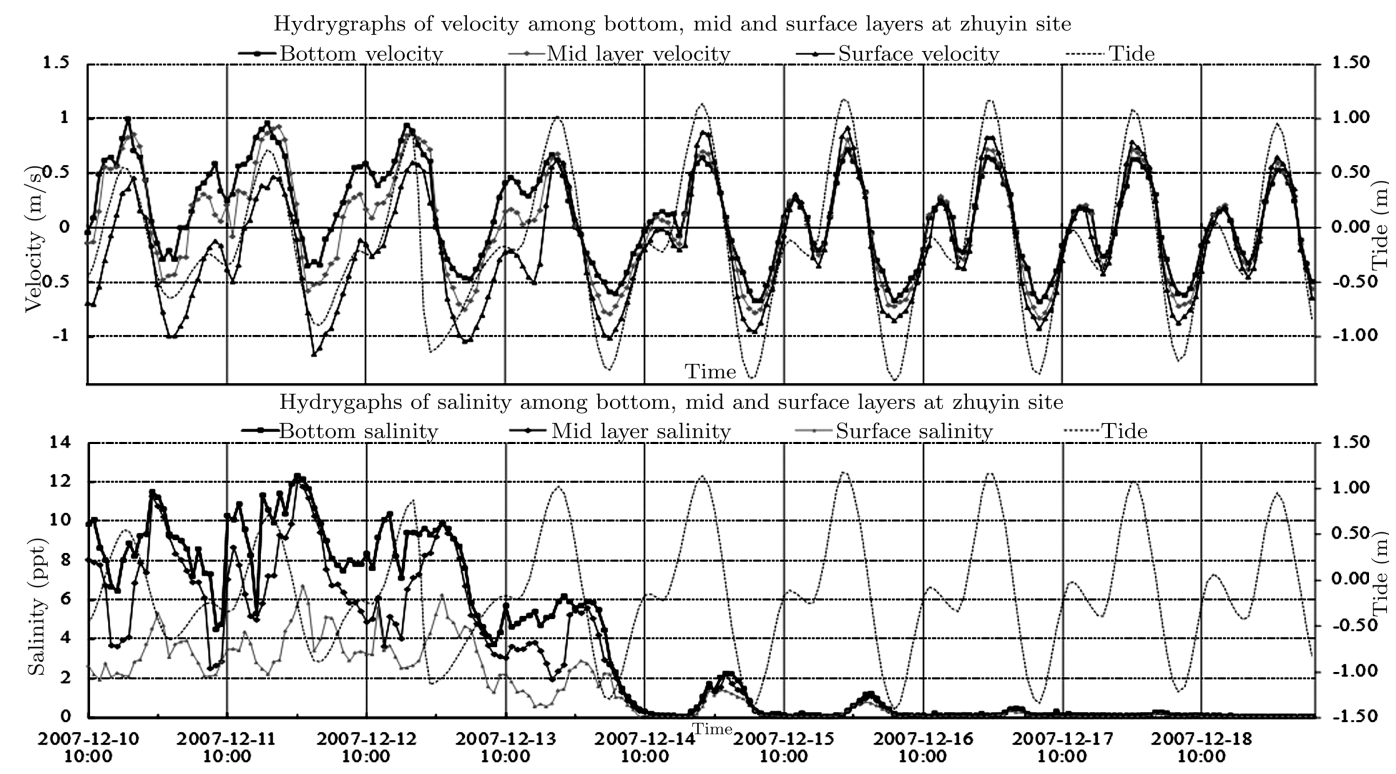

Figure 2: The hydrographs of velocity and salinity at Zhuyin station

From December 15 to 18, when salinity value in each layer and vertical salinity gradient are lower, the time of flood or ebb in each layer are almost the same. No matter during ebbing or flooding, the flow velocity monotonously decreases from water surface to the bottom, and the maximum velocity of ebb tide is bigger than that of flood in each layer, which is suggested by common sence. From December 11 to 12, when salinity value and vertical salinity gradient are higher, the maximum velocity of flood tide near the bottom water layer is as twice magnitude as that of the surface, and the flood duration of bottom water layer is about 9 hour longer than that of the surface. It means that the inflexional velocity profile with positive and negative velocity coexist can remain for about 9 hours. This is very different from most estuaries, in which the inflexional velocity profile usually occurs for only 20-30 minutes [3]. The flood duration 
near the bottom last amazingly for 15-18 hours one day, and the ebb duration only last for as short as 6 hours. In the mean time, the maximum flooding velocity and duration of surface water layer come to their minimum values in a spring-neap tidal circle, while the maximum ebbing velocity and duration come to their maximum values.

Based on the hourly flow rate data measured at Guadingjia (Figure 1) near the river mouth in 2005-2006, Bao and Liu(2009) studied the relationship between saltwater intrusion length in the estuary and river discharge [1]. They noticed that when the salinity overproof duration for water supply at the upstream increased swiftly from 7-16 hours to 24 hours per day, as a result of violent saltwater intrusion in two days, the average daily flow rate of Guadingjia was close to zero or minus, meaning a net landwards flow rates. In the mean time, a continuous stream of fresh water was still pouring into Modaomen channel from the upstream. This indicates that there indeed exists longtime upstream saltwater intrusion in the bottom water layer. It may be attributed to the particularity of terrain and upstream river flow discharge of this channel.

\section{CONCLUSION}

As the tidal range increases from neap to spring tide, the salinity in each water layer decreases unexpectedly. This peculiar phenomenon is attributed to the extraordinary flow process in Modaomen channel. When salinity value in each layer and vertical salinity gradient are lower during spring tide, the flow velocity monotonously decreases from water surface to the bottom. When salinity value in each layer and vertical salinity gradient are higher during neap tide, the flow velocity unexpectedly increases monotonously from water surface to the bottom during flood period, and flood duration at the bottom is surprisingly as long as $15-18$ hours. In addition, the inflexional velocity profile may remain amazingly for about 9 hours. This could be driven by baroclinic pressure under the condition of tides, topography and upstream runoff discharge of this channel.

\section{Acknowledgements}

We would like to give thanks to the financial support from the National Natural Science Foundation of China (11042003). Our sincere gratitude is especially delivered to Professor J. F. Zhou for his scrutinous suggestion of linguistic improvement.

\section{REFERENCES}

1. Bao Y, Liu J, Ren J, et al. Research of law and dynamic mechanism for strong saline water intrusion in modaomen Waterway (in Chinese). Science in China $(G), 2009 ; 39(10)$ : 1527-1534

2. Liu J, Bao Y, Huang Y. Contrast of movement law of salinity intrusion in Modaomen waterway in the wet year and the dry year (in Chinese). Chinese Journal Of Theoretical and Applied Mechanics, 2010; 42(6): 1098-1103

3. Liu H, Wu C Y, Bao Y, et al. Research on the Modaomen estuarine circulation under a winter monsoon (in chinese). The Ocean Engineering, 2008; 26(2): 102-111 